\title{
Application of multicolor banding combined with heterochromatic and locus-specific probes identify evolutionary conserved breakpoints in Hylobates pileatus
}

Wiwat Sangpakdee ${ }^{1,2}$, Alongklod Tanomtong ${ }^{2}$, Xiaobo Fan ${ }^{1}$, Krit Pinthong ${ }^{3}$, Anja Weise ${ }^{1}$ and Thomas Liehr ${ }^{*^{*}}$

\begin{abstract}
Background: The question what makes Homo sapiens sapiens (HSA) special among other species is one of the basic questions of mankind. A small contribution to answer this question is to study the chromosomal constitution of HSA compared to other, closely related species. In order to check the types and extent of evolutionary conserved breakpoints we studied here for the first time the chromosomes of Hylobates pileatus (HPI) compared to HSA and Hylobates lar (HLA) by means of molecular cytogenetics.

Results: Overall, 68 new evolutionary conserved breakpoints compared to HSA could be characterized in this study. Interestingly, only seven of those were different compared to HLA. However, application of heterochromatic human DNA-probes provided evidence that observed high chromosomal rearrangement rates of gibbons in HPI happened rather

in these repetitive elements than in euchromatin, even though most centromeric positions were preserved in HPI compared to HSA.

Conclusion: Understanding genomes of other species and comparing them to HSA needs full karyotypic and high resolution genomic data to approach both: euchromatic and heterochromatic regions of the studied chromosomecontent. This study provides full karyotypic data and previously not available data on heterochromatin-syntenies of HPI and HSA.
\end{abstract}

Keywords: Multicolor banding (MCB), Hylobates pileatus (HPI), Hylobates lar (HLA), Evolution, Centromeric positions, Heterochromatin

\section{Background}

Understanding evolution of human (Homo sapiens sapiens, HSA) is one of the major interests of man, latest since Charles Darwin published 'The origin of species by means of natural selection' [1]. As Hominoidea superfamily includes great apes (chimpanzees, bonobos, orangutans, gorillas, humans) as well as lesser apes (gibbons; Hylobatidae), studies on chromosomal evolution should include all groups of the superfamily HSA belongs to. For lesser apes it has been shown

\footnotetext{
* Correspondence: Thomas.Liehr@med.uni-jena.de

${ }^{1}$ Jena University Hospital, Friedrich Schiller University, Institute of Human Genetics, Kollegiengasse 10, D-07743 Jena, Germany

Full list of author information is available at the end of the article
}

previously that they underwent high numbers of chromosomal rearrangements including inversions, translocations, fissions and fusions [2] after they divided from their common ancestor with great apes around 15-20 million years ago $[3,4]$. The chromosomal rearrangement rate of gibbons was suggested to be at least an order of magnitude higher than the average rearrangement rate in mammals $[5,6]$, making them an interesting model of evolution.

The family Hylobatidae includes at least 12 species divided into four genera with different constitutional chromosome numbers: Hoolock $(2 \mathrm{n}=38)$, Hylobates $(2 \mathrm{n}=$ 44), Symphalangus $(2 \mathrm{n}=50)$ and Nomascus $(2 \mathrm{n}=52)$ [7]. Besides determining chromosomal numbers and doing 
basic cytogenetic studies, only a few lesser apes were studied in detail by molecular studies including fluorescence in situ hybridization (FISH). In Hylobates there were studied yet by FISH with a whole genomic view Hylobates lar (HLA) [8, 9], H. klossii [10], H. moloch [11], and $H$. pileatus (HPI) [11]. For $H$. muelleri, and $H$. agilis (HAG) it was only shown by now that alphasatellite sequences as present in HSA are not detectable in those species, including in HLA and HPI [9]. HAG was also studied using a few selected chromosome paints [12]. For $H$. albibarbis genetic studies were not done yet, which still could be helpful to solve the question if it is a subspecies of $H$. agilis [13]. Finally, there are FISH-studies on H. leucogenys (now called Nomascus leucogenys) [14], H. concolor (now called Nomascus concolor) [15], H. syndactylus (renamed to Symphalangus syndactylus) [16].

The pileated gibbon (HPI) has 44 chromosomes and was first karyotyped in 2007 [17]. Yet, detailed molecular cytogenetic characterization was not done, even though high throughput sequencing of its genome was performed recently [11]. Here we report the characterization of 68 evolutionary conserved breakpoints (ECBs) in HPI based on FISH applying high resolution multicolor banding (MCB), locus-specific probes and also all human repetitive probes apart from centromeric ones. The obtained data is an important addition to the already available molecular data.

\section{Results}

Results of the present study are summarized in Tables 1 and 2. Representative results of $\mathrm{MCB}$, as well as regions being homologous to acrocentric short arms in HSA, the NOR-region, the region 9p12/9q13 and 16q11.2 are shown in Fig. 1. Also one locus-specific probe based FISH result for a region being homologous in HPI to human chromosome 2 can be found there. The latter is shown, as this region/rearrangement could not be visualized by MCB.

As shown in Table 1, most HPI chromosomes are composed from regions being homologous to two or more HSA chromosomes; only HPI chromosomes X, Y, 14,17 and 20 are homologous to only one human chromosome, each. Of those only chromosomes $\mathrm{X}, \mathrm{Y}$ and 20 do not present gross chromosomal rearrangements according to FISH.

Table 1 Homologous regions, centromere position and heterochromatic inserts observed in HPI compared to HSA chromosomes

\begin{tabular}{|c|c|c|}
\hline HPI chr.\# & HPI-chromosomes reported as derivatives of human chromosomes & Centromeric position \\
\hline 1 & 2qter- > 2q22.3::7q21.11- > 7p22.3::7q21.11-> 7qter & as in HSA7 \\
\hline 2 & 6qter- > 6q15::6p10-> 6q15::4q13.1-> 4q10::4q13.1-> 4q26::10p12.1-> 10pter & as in HSAG \\
\hline 3 & het:3q22.1- > 3p12.3::3p14.3-> 3p12.3::13p13- > 13q21.32::13q33.2-> 13q21.32::13q33.2-> 13qter & as in HSA13 \\
\hline 4 & 10qter- > 10p12.1::4q26- > 4qter & as in HSA10 \\
\hline 5 & 16qter- > 16q22.2::5q31.1-> 5q14.1::16p12.2-> 16q22.2::5q31.1- > 5qter & as in HSA16 \\
\hline 6 & 18qter-> 18q10::18p11.32- > 18p10::11p10-> 11q13.1::1q23.1- > 1p31.1: & as in HSA11 / 18 \\
\hline 7 & 12pter- > 12p11.21::1q25.2- > 1q23.1::1p33- > 1p35.2::3p14.3- > 3p26.3::8p22-> 8pter & neo $12 \mathrm{p} 11.21 / 1 \mathrm{p} 35.3$ \\
\hline 8 & $\begin{array}{l}\text { 16pter- > 16p12.2::5q14.1- > 5p10::2p11.2- > 2q10::22p13- > 22q13.33::17p13.3- > 17p11.2::9p24.3- > } \\
\text { 9p12::9p24.3-> 9pter }\end{array}$ & as in HSA22 \\
\hline 9 & $: 17 q 22->17 q 23.2:: 17 q 21.1->17 q 10:: 17 q 21.1->17 q 22:: 9 q 21.12->9 p 12:: 9 q 21.12->9 q t e r$ & as in HSA9 \\
\hline 10 & 2pter- > 2p22.3::19q13.12-> 19q13.31::12q13.1-> 12p11.21::3q24- > 3qter & as HSA12 \\
\hline 11 & 1 pter- > 1p35.2::8q21.11- > 8p10::8q21.11- > 8qter & as in HSA8 \\
\hline 12 & $: 15 q 22.1->15 p 13:: 15 q 22.1->15 q 26.3:: 21 q 10->21 q t e r$ & as in HSA15 \\
\hline 13 & 19qter- > 19q13.42::12q22- > 12q13.3::19q13.12-> 19p13.2::19q13.32- > 19q13.42::12q22- > 12qter & as in HSA19 \\
\hline 14 & 11qter- > 11q13.1::11p15.5- > 11p11.2: & neo $11 q 13.1 / 11 p 15.5$ \\
\hline 15 & $: 2 q 22.3->2 q 14.2:: N O R:: 8 q 10->$ 8p22::3q22.1- > 3q24::12q13.3-> 12q13.1: & as in HSA8 \\
\hline 16 & 17qter- > 17q23.2::2q14.2- > 2q10::17p11.2- > 17q11.2::2p11.2- > 2p22.3::19q13.31- > 19q13.32::19p13.2- > 19pter & as in HSA17 \\
\hline 17 & $: 14 q 21.2->14 p 13:: 14 q 21.2->14 q$ ter & as in HSA14 \\
\hline 18 & :1q32.2-> 1q25.2::11p10-> 11p11.2::1p33-> 1p31.1::1q32.2-> 1qter & as in HSA11 \\
\hline 19 & 5pter- > 5q10::4p10- > 4pter - in centromere midi54+ & as HSA4 \\
\hline 20 & :6p21.2-> 6q10::6p21.2-> 6pter & as in HSAG \\
\hline 21 & 20pter- > 20qter in centromere midi54+ & as in HSA20 \\
\hline$x$ & Xpter- > Xqter & as in HSAX \\
\hline Y & Ypter-> Yqter & as in HSAY \\
\hline
\end{tabular}


Table 2 The 68 ECBs detected in this study in HPI given as cytoband and genomic data (GRCh37/hg19) as well as FISH-probes used and comparison to HLA

\begin{tabular}{|c|c|c|c|}
\hline Breakpoint (cytoband) & Narrowed down by & Localization acc. to GRCh37/hg19 & Same ECB in HLA [8] \\
\hline $1 p 35.2$ & RP4-669K10 / RP11-114B7 & $28,853,741-33,101,404$ & + \\
\hline $1 \mathrm{p} 33$ & RP11-330M19 / RP4-631H13 & $48,285,909-53,304,823$ & + \\
\hline $1 p 31.1$ & RP4-759M20 / RP5-944F13 & $67,033,180-70,103,142$ & + \\
\hline $1 \mathrm{q} 23.1$ & RP11-307C12 / RP11-343F16 & $154,965,587-164,006,044$ & + \\
\hline $1 \mathrm{q} 25.2$ & RP5-990P15 / RP11-181K3 & $178,490,946-183,063,114$ & + \\
\hline $1 \mathrm{q} 32.2$ & RP11-110E24 / RP11-434B7 & $210,211,975-213,224,588$ & + \\
\hline $2 p 22.3$ & RP11-23B13 / RP11-119B15 & $30,979,722-35,864,069$ & + \\
\hline $2 \mathrm{p} 11.2$ & RP11-316G9 / RP11-708D7 & $90,202,000-95,617,775$ & + \\
\hline $2 \mathrm{p} 11.1 \sim \mathrm{q} 11.1$ & n.a. & $90,500,000-96,800,000$ & - \\
\hline $2 q 14.2$ & Proximal to RP11-6906 & $\sim 110,000,000-121,987,648$ & + \\
\hline $2 q 22.3$ & RP11-107E5 / RP11-21M18 & $145,324,328-151,156,597$ & + \\
\hline $3 p 26.3$ & Proximal to subtel3pter & $\sim 2,000,000-\sim 5,000,000$ & + \\
\hline $3 p 14.3$ & RP11-904G16 / RP11-229A12 & $54,646,599-57,395,394$ & - \\
\hline $3 p 12.3$ & RP11-552N9 / RP11-16M12 & $72,550,809-78,313,071$ & + \\
\hline $3 q 22.1$ & RP11-221E20 / RP11-517B11 & $128,695,100-131,245,291$ & \\
\hline $3 q 24$ & RP11-88H10 7 RP11-500K7 & $145,702,132-147,840,000$ & \\
\hline $4 \mathrm{p} 11 \sim \mathrm{q} 11$ & n.a. & $48,200,000-52,700,000$ & + \\
\hline $4 q 13.1$ & RP11-498E11 / RP11-92H22 & $66,083,151-71,660,470$ & - \\
\hline $4 q 26$ & MCB & $126,000,000-137,000,000$ & + \\
\hline $5 p 11 \sim q 11.1$ & n.a. & $46,100,000-50,700,000$ & + \\
\hline $5 q 14.1$ & CTD-220003 / RP11-356D23 & $76,503,000-81,368,874$ & + \\
\hline $5 q 31.1$ & RP11-729C24 / CTD-2562E1 & $131,949,164-134,147,482$ & + \\
\hline $6 p 21.2$ & RP3-431A14 & $36,643,279-36,838,641$ & + \\
\hline $6 p 11.1 \sim q 11$ & n.a. & $58,700,000-63,300,000$ & + \\
\hline $6 q 15$ & RP11-223J24 / RP1-12208 & $86,460,000-90,317,182$ & + \\
\hline $7 p 22.3$ & Distal to subtel7pter & $0-\sim 2,500,000$ & + \\
\hline $7 q 21.11$ & RP11-235F21 / RP11-448A3 & $76,680,916-81,315,990$ & + \\
\hline $8 p 22$ & RP11-19N21 / RP11-459H21 & $16,574,193-21,137,316$ & + \\
\hline $8 p 11.1 \sim q 11.1$ & n.a. & $43,100,000-48,100,000$ & + \\
\hline $8 q 21.11$ & RP11-347D13 / RP11-48D4 & $70,768,835-77,707,273$ & + \\
\hline $9 p 24.3$ & Proximal to subtel9pter & $0-\sim 2,000,000$ & + \\
\hline $9 p 12$ & Proximal to RP11-128P23 & $35,360,000-\sim 50,000,000$ & + \\
\hline $9 q 21.12$ & Distal to RP11-373A9 & $72,849,245-\sim 90,000,000$ & + \\
\hline 10p12.1 & RP11-478H13 / RP11-379L21 & $23,279,768-29,095,050$ & + \\
\hline $11 \mathrm{p} 15.5$ & Distal to subtel1 1 pter & $0-\sim 1,200,000$ & + \\
\hline $11 \mathrm{p} 11.2$ & Distal to RP11-397M16 & $48,150,000-\sim 50,000,000$ & + \\
\hline $11 p 11.11-q 11$ & n.a. & $51,600,000-55,700,000$ & - \\
\hline $11 q 13.1$ & Distal to RP11-399J13 & $64,808,042-\sim 70,000,000$ & + \\
\hline $12 \mathrm{p} 11.21$ & RP11-517B23 & $31,471,644-32,029,051$ & - \\
\hline $12 q 13.1$ & Proximal to RP11-112N23 & $\sim 45,000,000-50,731,377$ & + \\
\hline $12 q 13.3$ & RP11-112N23 / RP11-629N8 & $50,912,474-65,153,301$ & + \\
\hline $12 q 22$ & RP11-24l19 / RP11-406H4 & $94,634,754-99,487,137$ & + \\
\hline
\end{tabular}


Table 2 The 68 ECBs detected in this study in HPI given as cytoband and genomic data (GRCh37/hg19) as well as FISH-probes used and comparison to HLA (Continued)

\begin{tabular}{|c|c|c|c|}
\hline $13 p 13$ & n.a. & $0-\sim 200,000$ & - \\
\hline $13 q 21.32$ & RP11-100C24 / RP11-187E23 & $57,831,960-67,194,978$ & - \\
\hline $13 q 33.2$ & RP11-564N10 / RP11-141M24 & $102,655,660-109,369,625$ & + \\
\hline $14 \mathrm{p} 13$ & n.a. & $0-\sim 200,000$ & - \\
\hline $14 q 21.2$ & RP11-35B20 / RP11-262M8 & $45,887,918-52,697,235$ & + \\
\hline $15 p 13$ & n.a. & $0-\sim 200,000$ & - \\
\hline $15 q 22.1$ & RP11-215J7 / RP11-219B17 & $55,027,961-60,973,768$ & + \\
\hline $15 q 26.3$ & Distal to subtel15qter & $\sim 102,200,000-102,531,392$ & + \\
\hline $16 p 12.2$ & RP11-705C1 & $24,089,175-24,270,169$ & - \\
\hline $16 q 22.2$ & RP5-991G20 / RP11-24I3 & $72,825,522-77,786,210$ & + \\
\hline $17 p 13.3$ & Proximal to subtel17pter & $0-\sim 500,000$ & + \\
\hline $17 p 11.2$ & Distal from RP11-746M1 & $\sim 15,000,000-21,160,776-28$ & + \\
\hline $17 q 11.2$ & Distal from RP11-403E9 & $28,495,981-\sim 40,000,000$ & - \\
\hline $17 q 21.1$ & RP11-47L3 / RP11-5809 & $33,661,870-38,501,211$ & + \\
\hline $17 q 22$ & RP5-843B9 / RP11-42901 & $46,228,000-50,467,875$ & + \\
\hline $17 q 23.2$ & RP11-142B17 / RP11-74H8 & $56,840,764-64,676,149$ & + \\
\hline $18 p 11.32$ & Proximal to subtel 18 pter & $0-\sim 200,000$ & + \\
\hline $18 p 11.1 \sim q 11.1$ & n.a. & $15,400,000-19,000,000$ & - \\
\hline 19p13.2 & RP11-565J3 / RP11-79F15 & $6,979,038-8,853,332$ & + \\
\hline $19 q 13.12$ & RP11-430N3 / RP11-649P22 & $36,673,365-38,450,859$ & + \\
\hline $19 q 13.31$ & RP11-537N4 / RP11-21J15 & $40,997,000-45,034,762$ & + \\
\hline $19 q 13.32$ & RP11-21J15 / RP11-1089K2 & $45,208,382-47,311,583$ & + \\
\hline $19 q 13.42$ & RP11-10l11 / RP11-44L20 & $51,622,674-53,472,377$ & + \\
\hline $21 \mathrm{p} 11.1 \sim q 11.1$ & n.a. & $10,900,000-14,300,000$ & + \\
\hline $22 p 13$ & n.a. & $0-\sim 200,000$ & - \\
\hline $22 q 13.33$ & Distal to subtel22qter & $\sim 51,000,000-51,304,566$ & + \\
\hline
\end{tabular}

In Table 2 the 68 detected ECBs are compared with previous results from HLA. Interestingly only 11/68 ECBs in HPI were different from HLA.

Application of all human repetitive probes for heterochromatic regions (apart from centromeric ones) revealed that there were no homologies present for sequences derived from human Yq12, 1q12, 9q12, and 15p12 11.2. However, the same heterochromatic sequences being present at HSA 16q11.2 (midi71) were detected at HPI 5, those in HSA 9p12/9q13 (midi36) as one block on HPI 9, those in HSA $19 \mathrm{p} 12 \sim 19 \mathrm{q} 12$ as one block on HPI 13, and those in human acrocentric short arms (midi54) on HPI 3, 8, 12, 17, 19, 21. NOR specific signals were only obtained as one block on both homologues of HPI 15. Finally, a block of HLA specific heterchromatin was present at end of HLA 3p.

The centromeric positions could be determined for all HPI chromosomes (Table 1). Interestingly, 20/23 positions are the same as in HSA. Centromeric positions of HSA chromosomes 11 and 18 are present at HPI 6 centromere. At HPI chromosomes 7 and 14 neocentromers are formed.

\section{Discussion}

Here the first molecular cytogenetic study in HPI is presented using FISH-banding, locus-specific and human heterochromatic probes. It could be confirmed that MCB is well suited to gain a genome wide view on ECBs rapidly also in a yet little studied species (for review on comparable studies see [18]) (Fig. 1). Further narrowing down of ECBs can then be easily done using selected locus-specific probes (Tables 1 and 2).

In general, HPI has, compared to human, a highly rearranged karyotype. However, considering changes like translocations and inversions in HLA, chromosomes of HPI are therefore less affected (see Table 2 and [8]). On the other hand heterochromatic regions underwent in HPI a much faster evolution than in HLA; direct comparison is only possible for the regions being present in HSA as acrocentric short arms (midi54-probe) [19]: on the one hand midi54-positive signals are detectable in HLA only at one spot, in HPI at 6 different locations. On the other hand in HLA this DNA amplified at a region homologous 


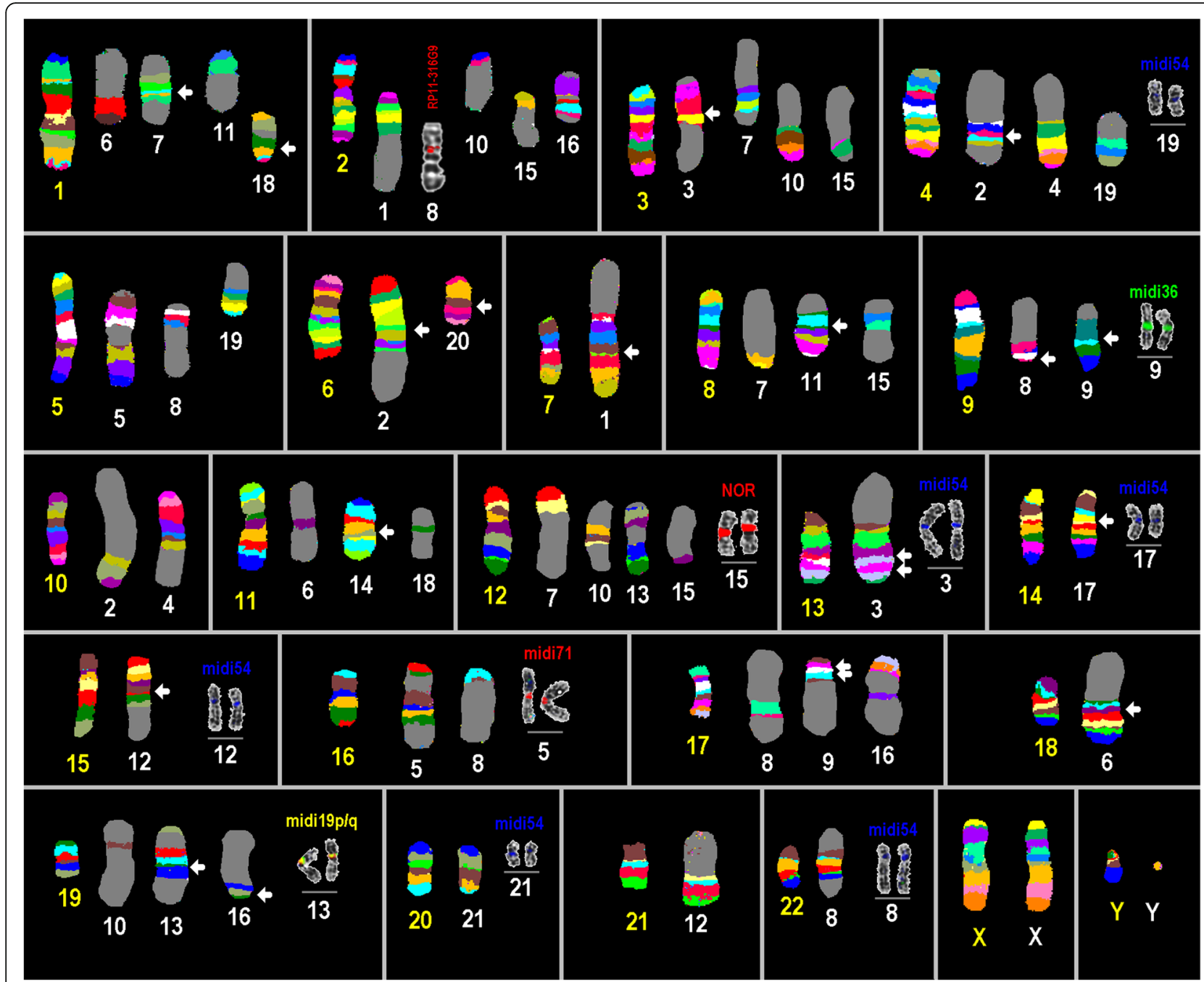

Fig. 1 Representative MCB results: HPI in comparison to HSA chromosomes are shown as pseudo-color results. HSA chromosomes are numbered by yellow figures, HPI chromosomes by white figures. The chromosomes are sorted according to the HSA-chromosomes. Additional FISHresults are shown also for corresponding HPI chromosomes, where necessary. Arrows highlight interchromosomal rearrangements. Abbreviations: midi = microdissection derived probe (consecutively numbered acc. to production or localization); NOR = nucleolus organizer region

to HSA 2, in HPI midi54-specific DNA seeded at regions homologous to centromeric positions of HSA chromosomes 4, 5, and all acrocentric short arms apart from HSA 21p. As homologous regions to HSA 15 and 21 are together on HPI chromosome 12, this also could be due to a fusion of both midi54-positive regions before an inversion in this chromosome took place.

At least 57/68 ECBs detected in HPI here are homologous to such detected in HLA. As 4/11 "new ECBs" compared to HLA are in terminal regions of HSA acrocentric short arms (Table 2), regions not present in HLA there, this may reduce the number of ECBs being really different in HPI and HLA to seven within the euchromatic sequences of HPI.

In this study the results obtained from application of heterochromatic probes suggest that chromosomal evolution in HPI was concentrated to these genomics regions, while in HLA euchromatin was hit more frequently. Both events might have been triggered by the recently suggested "propensity for a gibbon-specific retrotransposon (LAVA) to insert into chromosome segregation genes and alter transcription by providing a premature termination site, suggesting a possible molecular mechanism for the genome plasticity of the gibbon lineage" [8]. This, and the fact that centromeric positions changed in $13 / 23$ chromosomes in HLA [19] and only in $3 / 23$ in HPI needs to be elaborated by future studies.

\section{Conclusions}

Overall, this study highlights that to study and understand genomes of other species, e.g. in comparison to human, it is necessary first to have the karyotypic data 
and then to combine this with more sophisticated ones, like next generation sequencing data. The latter approach is due to technical restrictions principally not able to detect the peculiarities of heterochromatic regions, while molecular cytogenetic approaches cannot provide a basepair-resolution. Thus, only the combination of all available technical means will help us to understand the miracles of evolution and nature in the end.

\section{Methods}

\section{Cell culture and chromosomal preparation}

Immortalized lymphoblast cell lines derived from a male HPI, was provided by the Khon Kaen University, Thailand. Culture techniques as well as chromosome preparation followed standard protocols.

\section{Fluorescence in situ hybridization}

All protocols for FISH have been provided in our previous study by us in Fan et al. [18]; details on MCB, single and dual-color FISH techniques which were performed for the applied bacterial artificial chromosome (BAC-) probes and the commercially derived subtelomeric probes can be found there. All here used BAC-probes are listed in Additional file 1: Table S1. The homemade heterochromatin mix (HCM-) probe set [20] was also described there [18] covering chromosomal regions 1q12, 16q11.2 (midi71), 9q12, 9p12/ 9q13 (midi36) 15p11.2-p11.1, all acrocentric short arms (midi54), 19p12/q12 and Yq12.

\section{Additional file}

Additional file 1: The BAC probes applied in the present study. Abbreviations: n.a. $=$ not available; subtel = subtelomeric probe. (DOC $165 \mathrm{~kb})$

\section{Abbreviations}

ECBs: evolutionary conserved breakpoints; FISH: fluorescence in situ hybridization; H.: Hylobates; HAG: Hylobates agilis; HLA: Hylobates lar; HPI: Hylobates pileatus; HSA: Homo sapiens; MCB: multicolor banding; midi: microdissection derived probe; NOR: nucleolus organizer region.

\section{Competing interests}

The authors declare that they have no competing interests.

\section{Authors' contributions}

The study was planned by TL, AW and AT; cells were prepared by AW and AT; FISH was done by WS and XF, the article was drafted and worked out by TL. All authors read and approved final version.

\section{Acknowledgments \\ Supported in parts by the China Scholarship Council (support for FX), the DLR/BMBF RUS 09/008 (AW), and WS received financial support from the Thailand Research Fund through the Royal Golden Jubilee Ph.D. program joint funding with Khon Kaen University (Grant No. PHD/0081/2556).}

\section{Author details}

${ }^{1}$ Jena University Hospital, Friedrich Schiller University, Institute of Human Genetics, Kollegiengasse 10, D-07743 Jena, Germany. ${ }^{2}$ Department of Biology Faculty of Science, Khon Kaen University, 123 Moo 16 Mittapap Rd., Muang District, Khon Kaen 40002, Thailand. ${ }^{3}$ Faculty of Science and Technology,
Surindra Rajabhat University, 186 Moo 1, Maung District, Surin 32000, Thailand.

Received: 11 January 2016 Accepted: 10 February 2016

Published online: 17 February 2016

\section{References}

1. Darwin C. The origin of species by means of natural selection. https://www. andrew.cmu.edu/user/jksadegh/A\%20Good\%20Atheist\%20Secularist\%20 Skeptical\%20Book\%20Collection/Charles\%20Darwin\%20-\%20The\%20 Origin\%20of\%20Species\%20-\%206th\%20Edition.pdf. Accessed 24 Dec 2015.

2. Brown JD, O'Neill RJ. The mysteries of chromosome evolution in gibbons: methylation is a prime suspect. PLoS Genet. 2009;5:e1000501.

3. Müller S, Hollatz M, Wienberg J. Chromosomal phylogeny and evolution of gibbons (Hylobatidae). Hum Genet. 2013:113:493-501.

4. Perelman $P$, Johnson WE, Roos C, Seuánez HN, Horvath JE, Moreira MA, et al. A molecular phylogeny of living primates. PLOS Genet. 2011;7:e1001342.

5. Zhao S, Shetty J, Hou L, Delcher A, Zhu B, Osoegawa K, et al. Human, mouse, and rat genome large-scale rearrangements: stability versus speciation. Genome Res. 2004;14:1851-60.

6. Misceo D, Capozzi O, Roberto R, Dell'oglio MP, Rocchi M, Stanyon R, et al. Tracking the complex flow of chromosome rearrangements from the Hominoidea ancestor to extant Hylobates and Nomascus gibbons by high-resolution synteny mapping. Genome Res. 2008;18:1530-7.

7. Brandon-Jones D, Eudey AA, Geissmann T, Groves CP, Melnick DJ, Morales JC, et al. Asian primate classification. Int J Primatol. 2004;25:97-164.

8. Weise A, Kosyakova N, Voigt M, Aust N, Mrasek K, Löhmer S, et al. Comprehensive analyses of white-handed gibbon chromosomes enables access to 92 evolutionary conserved breakpoints compared to the human genome. Cytogenet Genome Res. 2015;145:42-9.

9. Baicharoen S, Miyabe-Nishiwaki T, Arsaithamkul V, Hirai Y, Duangsa-ard K, Siriaroonrat $\mathrm{B}$, et al. Locational diversity of alpha satellite DNA and intergeneric hybridization aspects in the Nomascus and Hylobates genera of small apes. PLoS One. 2014;9:e109151.

10. Arnold N, Stanyon R, Jauch A, O'Brien P, Wienberg J. Identification of complex chromosome rearrangements in the gibbon (Hylobates klossii) by flourescent in situ hybridization (FISH) of a human chromosome 2q specific microlibrary, yeast artificial chromosomes and reciprocal chromosome painting. Cytogenet Cell Genet. 1996;74:80-5.

11. Carbone L, Harris RA, Gnerre S, Veeramah KR, Lorente-Galdos B, Huddleston J, et al. Gibbon genome and the fast karyotype evolution of small apes. Nature. 2014:513(7517):195-201.

12. Hirai H, Mootnick AR, Takenaka O, Suryobroto B, Mouri T, Kamanaka Y, et al. Genetic mechanism and property of a whole-arm translocation (WAT) between chromosomes 8 and 9 of agile gibbons (Hylobates agilis). Chromosome Res. 2003;11:37-50.

13. RA Mittermeier, AB Rylands, DE Wilson. Handbook of the Mammals of the World. Vol 3: Primates, Lynx Edition, Barcelona 2013, pp 780-1.

14. Lee C, Rens W, Yang F. Multicolor fluorescence in situ hybridization (FISH) approaches for simultaneous analysis of the entire human genome. Curr Protoc Hum Genet. 2001; Chapter 4:Unit4.9.

15. Koehler U, Bigoni F, Wienberg J, Stanyon R. Genomic reorganization in the concolor gibbon (Hylobates concolor) revealed by chromosome painting. Genomics. 1995;30:287-92.

16. Koehler U, Arnold N, Wienberg J, Tofanelli S, Stanyon R. Genomic reorganization and disrupted chromosomal synteny in the siamang. (Hylobates syndactylus) revealed by fluorescence in situ hybridization. Am J Phys Anthropol. 1995;97:37-47.

17. Supanuam P, Tanomtong A, Khunsook S. Standardized karyotype and idiogram of the pileated gibbon, Hylobates pileatus (Primate, Hylobatidae) by G-banding and high-resolution technique. Cytologia. 2007;72:189-94.

18. Fan X, Pinthong K, Mkrtchyan H, Siripiyasing P, Kosyakova N, Supiwong W, et al. First detailed reconstruction of the karyotype of Trachypithecus cristatus (Mammalia: Cercopithecidae). Mol Cytogenet. 2013;6:58.

19. Mrasek K, Heller A, Rubtsov N, Trifonov V, Starke H, Claussen U, et al Detailed Hylobates lar karyotype defined by 25 -color FISH and multicolor banding. Int J Mol Med. 2003;12:139-46

20. Bucksch M, Ziegler M, Kosayakova N, Mulatinho MV, Llerena Jr JC, Morlot S, et al. A new multicolor fluorescence in situ hybridization probe set directed against human heterochromatin: HCM-FISH. J Histochem Cytochem. 2012;60:530-6. 